\title{
EXTRINSIC CIRCULAR TRAJECTORIES ON GEODESIC SPHERES IN A COMPLEX PROJECTIVE SPACE
}

Tuya BAO and Toshiaki ADACHI

\begin{tabular}{|c|l|}
\hline Citation & Osaka Journal of Mathematics. 54(4); 735-745 \\
\hline Issue Date & $2017-10$ \\
\hline Textversion & Publisher \\
\hline Right & CDepartments of Mathematics of Osaka University and Osaka City University. \\
\hline DOI & $10.18910 / 67011$ \\
\hline Is Identical to & https://doi.org//10.18910/67011 \\
\hline Relation & $\begin{array}{l}\text { The OJM has been digitized through Project Euclid platform } \\
\text { http://projecteuclid.org/ojm starting from Vol. 1, No. } 1 .\end{array}$ \\
\hline
\end{tabular}

\section{SURE: Osaka City University Repository}

https://dlisv03.media.osaka-cu.ac.jp/il/meta_pub/G0000438repository 


\title{
EXTRINSIC CIRCULAR TRAJECTORIES ON GEODESIC SPHERES IN A COMPLEX PROJECTIVE SPACE
}

\author{
TUYA BAO and Toshiaki ADACHI
}

(Received March 22, 2016, revised September 23, 2016)

\begin{abstract}
We say a trajectory for a Sasakian magnetic field on a geodesic sphere in a complex projective space to be extrinsic circular if it can be seen as a circle in the ambient space. We study how the moduli space of extrinsic circular trajectories behaves in the moduli space of all circles in the ambient complex projective space. As an application we characterize the geodesic sphere of special radius which lies on the boundary position of the family of Berger spheres among all geodesic spheres and that has a characteristic properties from the viewpoint of lengths of circles.
\end{abstract}

\section{Introduction}

A smooth curve $\sigma$ parameterized by its arclength on a Riemannian manifold $N$ is said to be a circle if there is a nonnegative constant $k$ and a field $Y$ of unit tangent vectors along $\sigma$ satisfying the system of differential equations $\nabla_{\dot{\sigma}} \dot{\sigma}=k Y, \nabla_{\dot{\sigma}} Y=-k \dot{\sigma}$. This constant $k$ is said to be the geodesic curvature of $\sigma$, and $\{\dot{\sigma}, Y\}$ to be its Frenet frame. Since circles of null geodesic curvature are geodesics, from the viewpoint of Frenet formula, there is no doubt that circles are simplest curve next to geodesics. On a real space form, which is one of a standard sphere, a Euclidean space and a real hyperbolic space, lengths of closed circles depend smoothly on their geodesic curvatures. But the situation is different on a complex projective space $\mathbb{C} P^{n}$. When we consider lengths of closed circles on $\mathbb{C} P^{n}$, circles whose Frenet frame forms a complex line at each point have different properties from others ([2] and see \$2). In order to explain this, in [4] the second author took trajectories on geodesic spheres in $\mathbb{C} P^{n}$, which are curves closely related with almost contact metric structures on these geodesic spheres. He considered them as curves in $\mathbb{C} P^{n}$, and showed that every circles on $\mathbb{C} P^{n}$ is obtained from some trajectory on some geodesic sphere.

In this paper we refine the study on trajectories which can be seen as circles in $\mathbb{C} P^{n}$ given in [4]. We investigate how the moduli space, the set of all congruence classes, of these trajectories on a given geodesic sphere is contained in the moduli space of circles in $\mathbb{C} P^{n}$. We find that circles of geodesic curvature $\sqrt{2 c} / 4$ in $\mathbb{C} P^{n}(c)$ of constant holomorphic sectional curvature $c$ have a singular property compared with other circles from the viewpoint of ex-

2010 Mathematics Subject Classification. Primary 53C22; Secondary 53C40, 53 B35.

The first author is partially supported by National Natural Science Foundation of China (No. 11561052) and by National Natural Science Foundation of China (No. 11661062).

The second author is partially supported by Grant-in-Aid for Scientific Research (C) (No. 24540075 \& No. 16K05126) Japan Society for the Promotion of Science. 
trinsic shapes of trajectories. These circles are also obtained as images of geodesics through a parallel isometric embedding $\left(S^{1} \times S^{n-1}\right) / \sim \rightarrow \mathbb{C} P^{n}$ given by Naitoh [14], and are characteristic circles from the viewpoint of length spectrum of circles (see §2 and [6]). As an application of our study we characterize a geodesic sphere $G\left(r_{0}\right)$ of special radius $r_{0}$ satisfying $\cot \left(\sqrt{c} r_{0} / 2\right)=1 / \sqrt{2}$ among real hypersurfaces in $\mathbb{C} P^{n}(c)$. It contains all these characteristic circles as trajectories. We note that geodesic spheres of radius satisfying $\cot (\sqrt{c} r / 2)<1 / \sqrt{2}$ are so-called Berger spheres (see [17]). Sectional curvatures of the geodesic sphere $G\left(r_{0}\right)$ lie in the interval $[K / 9, K]$ with $K=9 c / 8$, and the length of its shortest closed geodesic is $2 \pi / \sqrt{K}$. This geodesic can be seen as a circle in $\mathbb{C} P^{n}(c)$. Thus our result shows that not only the shortest closed geodesics but also trajectories on this geodesic sphere which can be seen as circles in $\mathbb{C} P^{n}$ have characteristic properties.

The authors are grateful to the referee who read their manuscript very carefully.

\section{Circles on a complex projective space}

In order to explain the background of our study, we shall start by recalling the moduli space of circles on a complex projective space $\mathbb{C} P^{n}$. For a circle $\sigma$ on $\mathbb{C} P^{n}$ which satisfies $\nabla_{\dot{\sigma}} \dot{\sigma}=k_{\sigma} Y, \nabla_{\dot{\sigma}} Y=-k_{\sigma} \dot{\sigma}$, we set $\tau_{\sigma}=\langle\dot{\sigma}, J Y\rangle$ with the complex structure $J$ on $\mathbb{C} P^{n}$, and call it the complex torsion of $\sigma$. Since $J$ is parallel, we see that $\tau_{\sigma}$ is constant along $\sigma$. We say two smooth curves $\sigma_{1}, \sigma_{2}$ on a Riemannian manifold $N$ which are parameterized by their arclengths to be congruent to each other if there is an isometry $\varphi$ of $N$ and a constant $t_{c}$ satisfying $\varphi \circ \sigma_{1}(t)=\sigma_{2}\left(t+t_{c}\right)$ for all $t$. It is known that circles on $\mathbb{C} P^{n}$ are classified into congruence classes by their geodesic curvatures and complex torsions (see [13]). Hence, the moduli space $\mathcal{M}\left(\mathbb{C} P^{n}\right)$ of circles of positive geodesic curvature on $\mathbb{C} P^{n}$, which is the set of all congruence classes of such circles, is set theoretically congruent to the band $(0, \infty) \times[0,1]$ in $\mathbb{R}^{2}$.

A smooth curve $\sigma$ parameterized by its arclength is said to be closed if there is a positive constant $t_{0}$ satisfying $\sigma\left(t+t_{0}\right)=\sigma(t)$ for all $t$. The minimum positive $t_{0}$ with this property is said to be the length of $\sigma$. For an open curve, a curve which is not closed, we consider that its length is infinity. Since two smooth curves which are congruent to each other have the same length, we can define a function $\mathcal{L}: \mathcal{M}\left(\mathbb{C} P^{n}(c)\right) \rightarrow(0, \infty]$ which shows lengths of circles. On $\mathbb{C} P^{n}(c)$, circles of geodesic curvature $\sqrt{2 c} / 4$ have characteristic properties. For $k>0$ and $\tau$ with $0 \leq \tau \leq 1$, we denote by $[\sigma(k, \tau)]$ the congruence class of circles of geodesic curvature $k$ and of complex torsion $\tau$ on $\mathbb{C} P^{n}(c)$. We put $\mathcal{M}_{k}=\{[\sigma(k, \tau)] \mid 0 \leq$ $\tau<1\}\left(\subset \mathcal{M}\left(\mathbb{C} P^{n}(c)\right)\right)$ for a positive $k$. Then we have an injective map $\Phi_{k}: \mathcal{M}_{k} \rightarrow \mathcal{M}_{\sqrt{2 c} / 4}$ given as $[\gamma(k, \tau)] \mapsto\left[\gamma\left(\sqrt{2 c} / 4,3 \sqrt{3} c k \tau\left(4 k^{2}+c\right)^{-3 / 2}\right)\right]$. This satisfies $\mathcal{L}=C_{k} \cdot \mathcal{L} \circ \Phi_{k}$ on $\mathcal{M}_{k}$ with $C_{k}=\sqrt{3 c /\left\{2\left(4 k^{2}+c\right)\right\}}$ (see [2]). Thus we have a lamination on $\mathcal{M}\left(\mathbb{C} P^{n}(c)\right)$ each of whose leaf is maximal with respect to the smooth property of $\mathcal{L}$ (see Fig. 1). Every leaf crosses to the set $\mathcal{M}_{\sqrt{2 c} / 4}$ of congruence classes of circles of geodesic curvature $\sqrt{2 c} / 4$.

\section{Trajectories for Sasakian magnetic fields}

Let $M$ be a real hypersurface in a Kähler manifold $\widetilde{M}$. On this hypersurface we have an almost contact metric structure induced by the complex structure $J$ on $\widetilde{M}$. If we denote by $\mathcal{N}$ a (local) unit normal vector field on $M$ in $\widetilde{M}$, this structure is a quartet $(\eta, \xi, \phi,\langle\rangle$, 


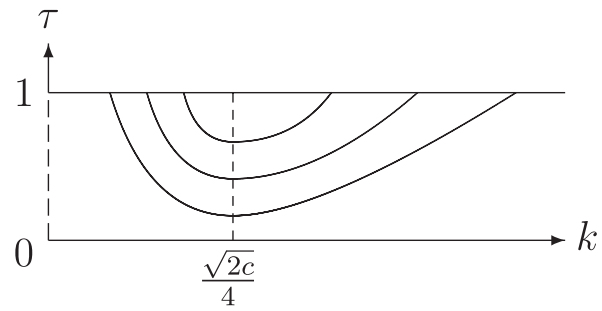

Fig. 1. Lamination on the moduli space of circles on $\mathbb{C} P^{n}(c)$

of a vector field $\xi$ defined by $\xi=-J \mathcal{N}$, a 1 -form $\eta$ given by $\eta(v)=\langle v, \xi\rangle$, a $(1,1)$-tensor $\phi$ defined by $\phi(v)=J v-\eta(v) \mathcal{N}$ and the metric $\langle$,$\rangle induced by the metric on \widetilde{M}$. These $\xi$ and $\phi$ are called the characteristic vector field and the characteristic tensor of $M$, respectively. We define a 2 -form $\mathbb{F}_{\phi}$ associated with this structure by $\mathbb{F}_{\phi}(v, w)=\langle v, \phi(w)\rangle$. One can easily find that it is a closed form (see [7]). Generally, a closed 2-form on a Riemannian manifold is said to be a magnetic field because it can be regarded as a generalization of a static magnetic field on a Euclidean 3-space $\mathbb{R}^{3}$ (see [15], for example). We therefore say that a constant multiple $\mathbb{F}_{\kappa}=\kappa \mathbb{F}_{\phi}(\kappa \in \mathbb{R})$ of the form $\mathbb{F}_{\phi}$, which is also closed, a Sasakian magnetic field on $M$.

A smooth curve $\gamma$ on $M$ which is parameterized by its arclength is said to be a trajectory for $\mathbb{F}_{\kappa}$ if it satisfies the differential equation $\nabla_{\dot{\gamma}} \dot{\gamma}=\kappa \phi(\dot{\gamma})$. When $\kappa=0$, which is the case that there is no influence of magnetic fields, trajectories are geodesics. Therefore, we may say that trajectories are extended objects of geodesics. To study properties of trajectories, we put $\rho_{\gamma}=\left\langle\dot{\gamma}, \xi_{\gamma}\right\rangle$, and call this function along a trajectory $\gamma$ its structure torsion. We here recall Gauss and Weingarten formulae. If we denote by $\widetilde{\nabla}$ the Riemannian connection on $\widetilde{M}$, they are given as

$$
\widetilde{\nabla}_{X} Y=\nabla_{X} Y+\left\langle A_{M} X, Y\right\rangle \mathcal{N} \text { and } \widetilde{\nabla}_{X} \mathcal{N}=-A_{M} X
$$

for vector fields $X, Y$ tangent to $M$. Here $A_{M}$ denotes the shape operator of $M$ in $\widetilde{M}$ with respect to $\mathcal{N}$. By these formulae we have $\nabla_{X} \xi=\phi A_{M} X$, hence we get

$$
\rho_{\gamma}^{\prime}=\left\langle\kappa \phi \dot{\gamma}, \xi_{\gamma}\right\rangle+\left\langle\dot{\gamma}, \phi A_{M} \dot{\gamma}\right\rangle=\frac{1}{2}\left\langle\dot{\gamma},\left(\phi A_{M}-A_{M} \phi\right) \dot{\gamma}\right\rangle,
$$

because $A_{M}$ is symmetric and $\phi$ is skew-symmetric. Therefore the structure torsion for each trajectory is a constant function if $A_{M}$ and $\phi$ are simultaneously diagonalizable.

We denote by $\iota: M \rightarrow \widetilde{M}$ an isometric immersion. For a curve $\gamma$ on $M$ we have a curve $\iota \circ \gamma$ on $\widetilde{M}$. We call this the extrinsic shape of $\gamma$, and denote it also by $\gamma$ for the sake of simplicity. In this paper we study extrinsic shapes of trajectories on a geodesic sphere $G(r)$ of radius $r(0<r<\pi / \sqrt{c})$ in $\mathbb{C} P^{n}(c)$. It is well known that the characteristic vector $\xi_{p}$ at each point $p \in M=G(r)$ is a principal curvature vector associated with $\delta_{M}=\sqrt{c} \cot (\sqrt{c} r)$, and that each tangent vector orthogonal to $\xi_{p}$ is a principal curvature vector associated with $\lambda_{M}=(\sqrt{c} / 2) \cot (\sqrt{c} r / 2)$. In particular, its shape operator and its characteristic tensor are simultaneously diagonalizable, hence each trajectory has constant structure torsion. By applying Gauss and Weingarten formulae, for a trajectory $\gamma$ for $\mathbb{F}_{K}$ on $G(r)$ we have 


$$
\begin{aligned}
\widetilde{\nabla}_{\dot{\gamma}} \dot{\gamma}= & \kappa \phi \dot{\gamma}+\left\{\lambda_{M}\left(1-\rho_{\gamma}^{2}\right)+\delta_{M} \rho_{\gamma}^{2}\right\} \mathcal{N}, \\
\widetilde{\nabla}_{\dot{\gamma}} \widetilde{\nabla}_{\dot{\gamma}} \dot{\gamma}= & \kappa J \widetilde{\nabla}_{\dot{\gamma}} \dot{\gamma}+\left\{\lambda_{M}\left(1-\rho_{\gamma}^{2}\right)+\delta_{M} \rho_{\gamma}^{2}-\kappa \rho_{\gamma}\right\} \widetilde{\nabla}_{\dot{\gamma}} \mathcal{N} \\
=- & \left\{\kappa^{2}\left(1-\rho_{\gamma}^{2}\right)+\left\{\lambda_{M}+\left(\delta_{M}-\lambda_{M}\right) \rho_{\gamma}^{2}\right\}^{2}\right\} \dot{\gamma} \\
& \quad+\left\{\lambda_{M}-\kappa \rho_{\gamma}+\left(\delta_{M}-\lambda_{M}\right) \rho_{\gamma}^{2}\right\}\left\{\kappa+\left(\delta_{M}-\lambda_{M}\right) \rho_{\gamma}\right\}\left(\rho_{\gamma} \dot{\gamma}-\xi_{\gamma}\right) .
\end{aligned}
$$

We shall call a curve on a real hypersurface extrinsic circular if its extrinsic shape is a circle of positive geodesic curvature. In view of the above computation we get the following.

Lemma 1. A trajectory $\gamma$ for $\mathbb{F}_{K}$ on a geodesic sphere $G(r)$ in $\mathbb{C} P^{n}(c)$ is extrinsic circular if and only if it satisfies one of the following conditions:

1) $\rho_{\gamma}= \pm 1$,

2) $\lambda_{M}-\kappa \rho_{\gamma}+\left(\delta_{M}-\lambda_{M}\right) \rho_{\gamma}^{2}=0$,

3) $\kappa+\left(\delta_{M}-\lambda_{M}\right) \rho_{\gamma}=0$.

Corresponding to each case, the geodesic curvature $k_{\gamma}$ and the complex torsion $\tau_{\gamma}$ of the extrinsic shape are as follows:

1) $k_{\gamma}=\delta_{M}, \tau_{\gamma}=\mp 1$,

2) $k_{\gamma}=|\kappa|, \tau_{\gamma}=-\operatorname{sgn}(\kappa)$,

3) $k_{\gamma}=\sqrt{\kappa^{2}-2 \lambda_{M} \kappa \rho_{\gamma}+\lambda_{M}^{2}}, \tau_{\gamma}=\left(2 \kappa \rho_{\gamma}^{2}-\kappa-\lambda_{M} \rho_{\gamma}\right) / k_{\gamma}$.

A geodesic sphere $G(r)$ in $\mathbb{C} P^{n}(c)$ of radius $r>(2 / \sqrt{c}) \sin ^{-1}(\sqrt{6} / 3)$ is known as a Berger sphere. Its sectional curvatures lie in the interval $[\epsilon K, K]$ with some $\epsilon \in(0,1 / 9)$, and it has closed geodesics of length less than $2 \pi / \sqrt{K}$. It is known that their structure torsions are \pm 1 . Hence extrinsic shapes of these geodesics are circles by Lemma 1 . We should note that every trajectory $\gamma$ of structure torsion \pm 1 for an arbitrary Sasakian magnetic field $\mathbb{F}_{\kappa}$ is a geodesic because $\phi(\dot{\gamma})=0$.

\section{Extrinsic circular trajectories in the moduli space of circles}

In the first and the second cases in Lemma 1, the absolute values of complex torsions of extrinsic shapes are 1 . We hence study the third case. For a geodesic sphere $M=G(r)$ in $\mathbb{C} P^{n}$, we denote by $\mathcal{E}(M)$ the moduli space of extrinsic circular trajectories of third type in Lemma 1 for some Sasakian magnetic field on $M$ (for congruency of trajectories for Sasakian magnetic fields, see [3]). Since isometries of $M$ is equivariant, that is for each isometry $\varphi$ of $M$ there is an isometry $\tilde{\varphi}$ of $\mathbb{C} P^{n}$ satisfying $\iota \circ \varphi=\tilde{\varphi} \circ \iota$ with an isometric immersion $\iota: M \rightarrow \mathbb{C} P^{n}$, we see that extrinsic shapes of two curves on $M$ are congruent to each other in $\mathbb{C} P^{n}$ if they are congruent to each other in $M$. Therefore we have a map of $\mathcal{E}(M)$ into the moduli space $\mathcal{M}\left(\mathbb{C} P^{n}(c)\right)$ of circles of positive geodesic curvature. From now on we use $\mathcal{E}(M)$ together with its image through this map.

First we study how $\mathcal{E}(M)$ is included in $\mathcal{M}\left(\mathbb{C} P^{n}(c)\right)$. As we have $\delta_{M}-\lambda_{M}=-c /\left(4 \lambda_{M}\right)$, in the case that $4 \kappa \lambda_{M}=c \rho_{\gamma}$, we see by Lemma 1 that the geodesic curvature and the complex torsion of the extrinsic shape of circular trajectory $\gamma$ are expressed as

$$
k_{\gamma}=\sqrt{\lambda_{M}^{2}-\frac{c \rho_{\gamma}^{2}}{2}+\frac{c^{2} \rho_{\gamma}^{2}}{16 \lambda_{M}^{2}}}, \quad \tau_{\gamma}=\frac{\rho_{\gamma}\left(2 c \rho_{\gamma}^{2}-c-4 \lambda_{M}^{2}\right)}{4 \lambda_{M} k_{\gamma}} .
$$


When $\lambda_{M}=\sqrt{2 c} / 4$, we have by (4.1) that $k_{\gamma}=\lambda_{M}$ and $\tau_{\gamma}=4 \rho_{\gamma}^{3}-3 \rho_{\gamma}$. If we vary the parameter of structure torsion $\rho_{\gamma}$ in the interval $[-1,1]$ we find $\tau_{\gamma}$ takes all the values in the interval $[-1,1]$. In this case, we find that the map of $\mathcal{E}(M)$ into $\mathcal{M}\left(\mathbb{C} P^{n}(c)\right)$ is three to one on the image. When $\lambda_{M} \neq \sqrt{2 c} / 4$, the first equality of (4.1) shows that the map of $\mathcal{E}(M)$ into $\mathcal{M}\left(\mathbb{C} P^{n}(c)\right)$ is one to one onto the image, hence we may consider that $\mathcal{E}(M)$ is the subset of $\mathcal{M}\left(\mathbb{C} P^{n}(c)\right)$. In this case, by substituting the first equality of (4.1) to the second, we have

$$
\tau_{\gamma}^{2}=\frac{\left(k_{\gamma}^{2}-\lambda_{M}^{2}\right)\left(32 \lambda_{M}^{2} k_{\gamma}^{2}+4 c \lambda_{M}^{2}-c^{2}\right)^{2}}{c\left(c-8 \lambda_{M}^{2}\right)^{3} k_{\gamma}^{2}} .
$$

Here, as $\left|\rho_{\gamma}\right| \leq 1$, the first equality of (4.1) shows that

$$
\begin{cases}\lambda_{M} \leq k_{\gamma} \leq c /\left(4 \lambda_{M}\right)-\lambda_{M}\left(=-\delta_{M}\right), & \text { when } \lambda_{M}<\sqrt{2 c} / 4, \\ c /\left(4 \lambda_{M}\right)-\lambda_{M} \leq k_{\gamma} \leq \lambda_{M}, & \text { when } \sqrt{2 c} / 4<\lambda_{M}<\sqrt{c} / 2, \\ \lambda_{M}-c /\left(4 \lambda_{M}\right) \leq k_{\gamma} \leq \lambda_{M}, & \text { when } \lambda_{M} \geq \sqrt{c} / 2 .\end{cases}
$$

We consider the right hand side of (4.2) as a function $g(K)=g\left(K ; \lambda_{M}\right)$ on $K=k_{\gamma}^{2}$. We then have

$$
\frac{d g}{d K}=\frac{\lambda_{M}^{2}(8 K-c)\left(8 K+c-4 \lambda_{M}^{2}\right)\left(32 \lambda_{M}^{2} K+4 c \lambda_{M}^{2}-c^{2}\right)}{c\left(c-8 \lambda_{M}^{2}\right)^{3} K^{2}} .
$$

Hence we find the following.

1) When $\lambda_{M}<\sqrt{2 c} / 4$, the function $g$ is monotone increasing with respect to $K$ in the intervals $\left[\lambda_{M}^{2}, c / 8\right] \cup\left[\left(c\left(c-4 \lambda_{M}^{2}\right) /\left(32 \lambda_{M}^{2}\right),\left(-\delta_{M}\right)^{2}\right]\right.$, and is monotone decreasing in the interval $\left[c / 8,\left(c\left(c-4 \lambda_{M}^{2}\right) /\left(32 \lambda_{M}^{2}\right)\right]\right.$. We have

$$
g\left(\lambda_{M}^{2}\right)=g\left(\frac{c\left(c-4 \lambda_{M}^{2}\right)}{32 \lambda_{M}^{2}}\right)=0, \quad g\left(\frac{c}{8}\right)=g\left(\left(-\delta_{M}\right)^{2}\right)=1 .
$$

2) When $\sqrt{2 c} / 4<\lambda_{M}<\sqrt{c} / 2$, the function $g$ is monotone decreasing with respect to $K$ in the intervals $\left[\left(-\delta_{M}\right)^{2}, c\left(c-4 \lambda_{M}^{2}\right) /\left(32 \lambda_{M}^{2}\right)\right] \cup\left[c / 8, \lambda_{M}^{2}\right]$, and is monotone increasing in the interval $\left[c\left(c-4 \lambda_{M}^{2}\right) /\left(32 \lambda_{M}^{2}\right), c / 8\right]$.

3) When $\sqrt{c} / 2 \leq \lambda_{M}<\sqrt{2 c} / 2$, the function $g$ is monotone decreasing with respect to $K$ in the intervals $\left.\left[\delta_{M}^{2},\left(4 \lambda_{M}^{2}-c\right)\right) / 8\right] \cup\left[c / 8, \lambda_{M}^{2}\right]$, and is monotone increasing in the interval $\left.\left[\left(4 \lambda_{M}^{2}-c\right)\right) / 8, c / 8\right]$. We have

$$
g\left(\frac{4 \lambda_{M}^{2}-c}{8}\right)=\frac{\left(4 \lambda_{M}^{2}+c\right)^{3}\left(4 \lambda_{M}^{2}-c\right)}{c\left(8 \lambda_{M}^{2}-c\right)^{3}}
$$

which satisfies $0 \leq g\left(\left(4 \lambda_{M}^{2}-c\right) / 8\right)<1$ and is monotone increasing with respect to $\lambda_{M}$.

4) When $\lambda_{M} \geq \sqrt{2 c} / 2$, the function $g$ is monotone decreasing with respect to $K$ in the interval $\left[\delta_{M}^{2}, \lambda_{M}^{2}\right]$.

Thus for $M=G(r)$, the moduli space $\mathcal{E}(M)$ in $\mathcal{M}\left(\mathbb{C} P^{n}(c)\right)$ is like the following figures (Figs. $2-5)$ corresponding to $\lambda_{M}=(\sqrt{c} / 2) \cot (\sqrt{c} r / 2)$.

Next we study the behavior of $\mathcal{E}(G(r))$ when we vary the radius $r$. We consider the right hand side of (4.2) as a function $h(\Lambda)=h\left(\Lambda ; k_{\gamma}\right)$ on $\Lambda=\lambda_{M}^{2}$. We then have 


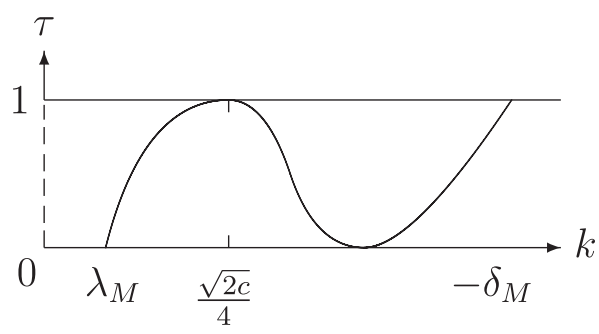

Fig. 2. $\lambda_{M}<\sqrt{2 c} / 4$

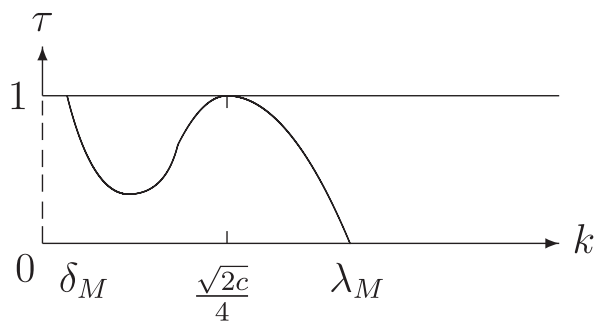

Fig. 4. $\sqrt{c} / 2 \leq \lambda_{M}<\sqrt{2 c} / 2$

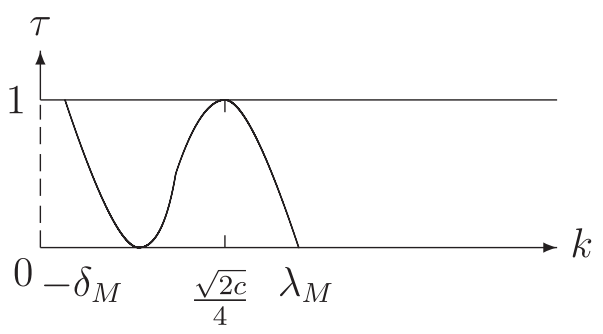

Fig. 3. $\sqrt{2 c} / 4<\lambda_{M}<\sqrt{c} / 2$

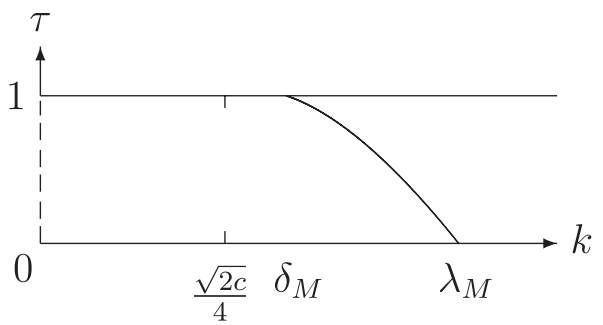

Fig. 5. $\lambda_{M} \geq \sqrt{2 c} / 2$

$$
\frac{d h}{d \Lambda}=\frac{\left(8 k_{\gamma}^{2}-c\right)^{2}\left(32 k_{\gamma}^{2} \Lambda+4 c \Lambda-c^{2}\right)(4 \Lambda+c)}{c(8 \Lambda-c)^{4} k_{\gamma}^{2}} .
$$

When $\Lambda>c / 4$, as $32 k_{\gamma}^{2} \Lambda+4 c \Lambda-c^{2}>0$, we have $d h / d \Lambda>0$, and when $\Lambda<c / 4$ we see $d h / d \Lambda$ changes its signature at $\Lambda=c^{2} /\left(32 k_{\gamma}^{2}+4 c\right)$. That is, when $\lambda_{M}>\sqrt{c} / 2$, the function $h\left(\Lambda ; k_{\gamma}\right)$ is increasing with respect to $\Lambda$ for each $k_{\gamma}$, and when $\lambda_{M}<\sqrt{c} / 2$ and $\lambda_{M} \neq \sqrt{2 c} / 4$, there is $k_{*}$ satisfying that $h\left(\Lambda ; k_{\gamma}\right)$ is decreasing with respect to $\Lambda$ if $k_{\gamma}<k_{*}$ and is increasing with respect to $\Lambda$ if $k_{\gamma}>k_{*}$. As we have $\lambda_{M}^{2}=c^{2} /\left(32 k_{*}^{2}+4 c\right)$, we see $k_{*}>\sqrt{2 c} / 4$ when $\lambda_{M}<\sqrt{2 c} / 4$ and $k_{*}<\sqrt{2 c} / 4$ when $\sqrt{2 c} / 4<\lambda_{M}<\sqrt{c} / 2$. Since $\cot (\sqrt{c} r / 2)$ is monotone decreasing with respect to $r$, if we take two geodesic spheres $M=$ $G\left(r_{1}\right), M^{\prime}=G\left(r_{2}\right)\left(r_{1}>r_{2}\right)$ so that the difference $r_{1}-r_{2}$ of their radii is sufficiently small, their moduli curves $\mathcal{E}(M), \mathcal{E}\left(M^{\prime}\right)$ of extrinsic circular trajectories are as Figs. 6 - 9 .

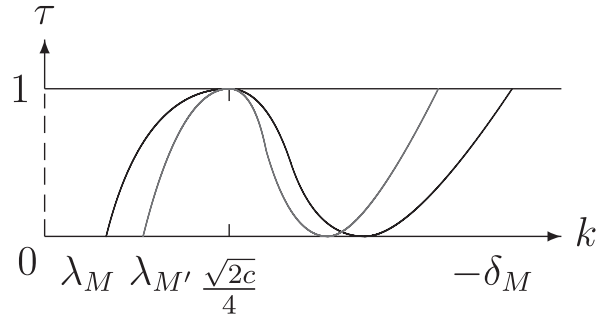

Fig. 6. $\lambda_{M}<\lambda_{M^{\prime}}<\sqrt{2 c} / 4$

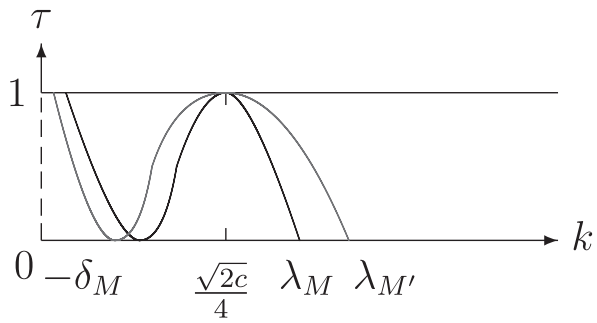

Fig.7. $\sqrt{2 c} / 4<\lambda_{M}<\lambda_{M^{\prime}}<\sqrt{c} / 2$

We divide the moduli space $\mathcal{M}\left(\mathbb{C} P^{n}(c)\right)$ into three subsets $\mathcal{M}_{-}\left(\mathbb{C} P^{n}\right) \cup \mathcal{M}_{\sqrt{2 c} / 4} \cup \mathcal{M}_{+}\left(\mathbb{C} P^{n}\right)$, where $\mathcal{M}_{-}\left(\mathbb{C} P^{n}\right)$ is the moduli space of circles of geodesic curvature less than $\sqrt{2 c} / 4$, and $\mathcal{M}_{+}\left(\mathbb{C} P^{n}\right)$ is the moduli space of circles of geodesic curvature greater than $\sqrt{2 c} / 4$. For each $k$ with $k<\sqrt{2 c} / 4$, if we vary $\lambda$ in the interval $(0, k]$, we see $\tau^{2}(\lambda, k)=\left(k^{2}-\lambda^{2}\right)\left(32 k^{2} \lambda^{2}+\right.$ $\left.4 c \lambda^{2}-c^{2}\right)^{2} /\left\{c\left(c-8 \lambda^{2}\right)^{3} k^{2}\right\}$ varies monotone decreasingly in the interval $[0,1)$. Hence 


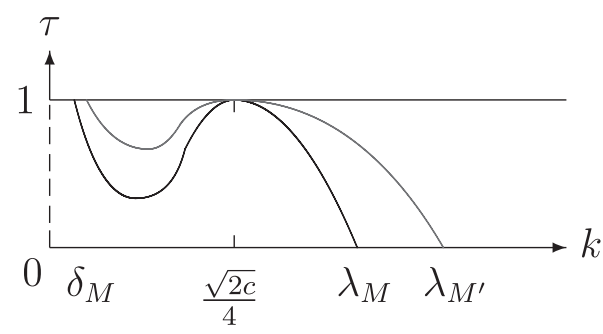

Fig. 8. $\sqrt{c} / 2 \leq \lambda_{M}<\lambda_{M^{\prime}}<\sqrt{2 c} / 2$

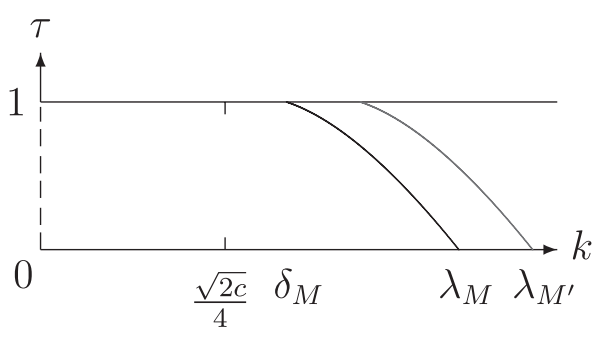

Fig.9. $\lambda_{M^{\prime}}>\lambda_{M} \geq \sqrt{2 c} / 2$

$\left\{\mathcal{E}(M) \cap \mathcal{M}_{-}\left(\mathbb{C} P^{n}\right) \mid \lambda_{M}<\sqrt{2 c} / 4\right\}$ is a foliation of $\mathcal{M}_{-}\left(\mathbb{C} P^{n}\right) \backslash\{[\gamma(k, 1)] \mid k<\sqrt{2 c} / 4\}$. If we vary $\lambda$ in the interval $\left[\left(-k+\sqrt{k^{2}+c}\right) / 2, c /\left\{2 \sqrt{8 k^{2}+c}\right\}\right]$, we see $\tau^{2}(\lambda, k)$ varies monotone decreasingly in the interval $[0,1]$. Hence $\left\{\mathcal{E}(M) \cap \mathcal{M}_{-}\left(\mathbb{C} P^{n}\right) \mid \sqrt{2 c} / 4<\lambda_{M}<\sqrt{2 c} / 2\right\}$ covers $\mathcal{M}_{-}\left(\mathbb{C} P^{n}\right)$. On the other hand, for each $k$ with $k>\sqrt{2 c} / 4$, if we vary $\lambda$ in the interval $\left[k,\left(k+\sqrt{k^{2}+c}\right) / 2\right]$, we see $\tau^{2}(\lambda, k)$ varies monotone increasingly in the interval $[0,1]$. Hence $\left\{\mathcal{E}(M) \cap \mathcal{M}_{+}\left(\mathbb{C} P^{n}\right) \mid \lambda_{M}>\sqrt{2 c} / 4\right\}$ is a foliation of $\mathcal{M}_{+}\left(\mathbb{C} P^{n}\right)$. If we vary $\lambda$ in the interval $(0, k]$, we see $\tau^{2}(\lambda, k)$ takes all the values in the interval $[0,1]$. Hence $\left\{\mathcal{E}(M) \cap \mathcal{M}_{+}\left(\mathbb{C} P^{n}\right) \mid \lambda_{M}<\sqrt{2 c} / 4\right\}$ covers $\mathcal{M}_{+}\left(\mathbb{C} P^{n}\right)$. Summarizing up we get the following.

Theorem 1. (1) The family $\left\{\mathcal{E}(G(r)) \cap \mathcal{M}_{-}\left(\mathbb{C} P^{n}\right) \mid r>(2 / \sqrt{c}) \sin ^{-1} \sqrt{2 / 3}\right\}$ forms a foliation of $\mathcal{M}_{-}\left(\mathbb{C} P^{n}\right) \backslash\{[\gamma(k, 1)] \mid k<\sqrt{2 c} / 4\}$.

(2) The family $\left\{\mathcal{E}(G(r)) \cap \mathcal{M}_{+}\left(\mathbb{C} P^{n}\right) \mid r>(2 / \sqrt{c}) \sin ^{-1} \sqrt{2 / 3}\right\}$ covers $\mathcal{M}_{+}\left(\mathbb{C} P^{n}\right)$.

(3) $\mathcal{E}\left(G\left((2 / \sqrt{c}) \sin ^{-1} \sqrt{2 / 3}\right)\right)=\mathcal{M}_{\sqrt{2 c} / 4}$.

(4) The family $\left\{\mathcal{E}(G(r)) \cap \mathcal{M}_{+}\left(\mathbb{C} P^{n}\right) \mid r<(2 / \sqrt{c}) \sin ^{-1} \sqrt{2 / 3}\right\}$ forms a foliation of $\mathcal{M}_{+}\left(\mathbb{C} P^{n}\right)$.

(5) The family $\left\{\mathcal{E}(G(r)) \cap \mathcal{M}_{-}\left(\mathbb{C} P^{n}\right) \mid \frac{2}{\sqrt{c}} \sin ^{-1} \frac{1}{\sqrt{3}}<r<\frac{2}{\sqrt{c}} \sin ^{-1} \sqrt{\frac{2}{3}}\right\}$ covers $\mathcal{M}_{-}\left(\mathbb{C} P^{n}\right)$.

\section{A characterization of the geodesic sphere}

In the previous section we see that in $\mathbb{C} P^{n}(c)$ geodesic spheres of radius $r=$ $(2 / \sqrt{c}) \sin ^{-1} \sqrt{2 / 3}$ have a specific property on extrinsic circular trajectories. In this section we give its characterizations among real hypersurfaces in $\mathbb{C} P^{n}$ from this point of view.

We take a trajectory $\gamma$ for $\mathbb{F}_{K}$ on a general real hypersurface $M$ of a Kähler manifold $\widetilde{M}$. By use of Gauss and Weingarten formulae, its extrinsic shape satisfies

$$
\begin{gathered}
\widetilde{\nabla}_{\dot{\gamma}} \dot{\gamma}=\kappa \phi \dot{\gamma}+\left\langle A_{M} \dot{\gamma}, \dot{\gamma}\right\rangle \mathcal{N}=\kappa J \dot{\gamma}+\left(\left\langle A_{M} \dot{\gamma}, \dot{\gamma}\right\rangle-\kappa \rho\right) \mathcal{N}, \\
\widetilde{\nabla}_{\dot{\gamma}} \widetilde{\nabla}_{\dot{\gamma}} \dot{\gamma}=-\kappa^{2} \dot{\gamma}-\left(\left\langle A_{M} \dot{\gamma}, \dot{\gamma}\right\rangle-\kappa \rho\right)\left(A_{M} \dot{\gamma}+\kappa \xi\right)+\frac{d}{d t}\left(\left\langle A_{M} \dot{\gamma}, \dot{\gamma}\right\rangle-\kappa \rho\right) \mathcal{N} .
\end{gathered}
$$

Thus, if the extrinsic shape of $\gamma$ is a circle of geodesic curvature $k_{\gamma}$, as we have $\widetilde{\nabla}_{\dot{\gamma}} \widetilde{\nabla}_{\dot{\gamma}} \dot{\gamma}=$ $-k_{\gamma}^{2} \dot{\gamma}$, we obtain the following by (5.1) and by comparing (5.2) with this equality:

$$
\begin{gathered}
k_{\gamma}^{2}=\kappa^{2}\left(1-\rho_{\gamma}^{2}\right)+\left\langle A_{M} \dot{\gamma}, \dot{\gamma}\right\rangle^{2}, \\
\left(k_{\gamma}^{2}-\kappa^{2}\right) \dot{\gamma}=\left(\left\langle A_{M} \dot{\gamma}, \dot{\gamma}\right\rangle-\kappa \rho_{\gamma}\right)\left(A_{M} \dot{\gamma}+\kappa \xi\right) .
\end{gathered}
$$

A real hypersurface $M$ is said to be Hopf if its characteristic vector field $\xi$ is principal at each point of $M$. It is known that the principal curvature associated with the characteristic 
vector field of a Hopf real hypersurface in $\mathbb{C} P^{n}$ is locally constant ([10]). We here consider the following condition at $p \in M$ :

(ET) The extrinsic shape of a trajectory $\gamma_{0}$ for some Sasakian magnetic field $\mathbb{F}_{\kappa_{0}}$ with $\dot{\gamma}_{0}(0)=\xi_{p}$ is a circle of geodesic curvature $k_{\gamma_{0}} \neq\left|\kappa_{0}\right|$.

Theorem 2. A connected real hypersurface $M$ in a complex projective space $\mathbb{C} P^{n}(c)$ of constant holomorphic sectional curvature $c$ is locally congruent to the geodesic sphere of radius $r=(2 / \sqrt{c}) \sin ^{-1} \sqrt{2 / 3}$ if and only if the following conditions hold:

i) At each point of $p \in M$,

a) the condition (ET) holds,

b) there exist constants $\kappa_{p}, \rho_{p}$ with $\kappa_{p} \neq 0, \sqrt{2 c} / 4$ and $\left|\rho_{p}\right|<1$ and linearly independent unit tangent vectors $v_{1}, \ldots, v_{2 n-2} \in U_{p} M$ with $\left\langle v_{i}, \xi_{p}\right\rangle=\rho_{p}$ which satisfy that the extrinsic shapes of trajectories $\gamma_{i}(i=1, \ldots, 2 n-2)$ for $\mathbb{F}_{\kappa_{p}}$ with $\dot{\gamma}_{i}(0)=v_{i}$ are circles of geodesic curvature $k_{i} \neq\left|\kappa_{p}\right|$;

ii) There is a trajectory whose extrinsic shape is a circle of geodesic curvature $\sqrt{2 c} / 4$ and of complex torsion $\tau \neq \pm 1$.

Proof. We are enough to show the "if" part. By the first condition, we have from (5.3) and (5.4) that

$$
k_{\gamma_{0}}=\left|\left\langle A_{M} \dot{\gamma}, \dot{\gamma}\right\rangle\right| \quad \text { and } \quad k_{\gamma_{0}}^{2} \xi_{p}=\left(\left\langle A_{M} \xi_{p}, \xi_{p}\right\rangle-\kappa_{0}\right) A_{M} \xi_{p}+\kappa_{0}\left\langle A_{M} \xi_{p}, \xi_{p}\right\rangle \xi_{p} .
$$

As $k_{\gamma_{0}} \neq\left|\kappa_{0}\right|$, we find that $\xi_{p}$ is principal. We denote by $\delta_{p}$ the principal curvature associated with $\xi_{p}$, which is locally constant.

By the second condition we decompose the both sides of (5.4) to components parallel to $\xi_{p}$ and orthogonal to it, and get the following:

$$
\begin{aligned}
\left(k_{i}^{2}-\kappa_{p}^{2}\right) \rho_{p} & =\left(\left\langle A_{M}\left(v_{i}-\rho_{p} \xi_{p}\right), v_{i}-\rho_{p} \xi_{p}\right\rangle+\delta_{p} \rho_{p}^{2}-\kappa_{p} \rho_{p}\right)\left(\rho_{p} \delta_{p}+\kappa_{p}\right), \\
\left(k_{i}^{2}-\kappa_{p}^{2}\right)\left(v_{i}-\rho_{p} \xi_{p}\right) & =\left(\left\langle A_{M}\left(v_{i}-\rho_{p} \xi_{p}\right), v_{i}-\rho_{p} \xi_{p}\right\rangle+\delta_{p} \rho_{p}^{2}-\kappa_{p} \rho_{p}\right) A_{M}\left(v_{i}-\rho_{p} \xi_{p}\right) .
\end{aligned}
$$

Since $k_{i} \neq\left|\kappa_{p}\right|$, by (5.6) we find that $v_{i}-\rho_{p} \xi_{p}$ is principal. We denote by $\alpha_{i}$ the principal curvature of $v_{i}-\rho_{p} \xi_{p}$. Then (5.5) and (5.6) turn to

$$
\begin{aligned}
\left(k_{i}^{2}-\kappa_{p}^{2}\right) \rho_{p} & =\left\{\alpha_{i}\left(1-\rho_{p}^{2}\right)+\rho_{p}^{2} \delta_{p}-\kappa_{p} \rho_{p}\right\}\left(\rho_{p} \delta_{p}+\kappa_{p}\right), \\
k_{i}^{2}-\kappa_{p}^{2} & =\left\{\alpha_{i}\left(1-\rho_{p}^{2}\right)+\rho_{p}^{2} \delta_{p}-\kappa_{p} \rho_{p}\right\} \alpha_{i} .
\end{aligned}
$$

Hence we have $\rho_{p} \alpha_{i}=\rho_{p} \delta_{p}+\kappa_{p}$. If $\rho_{p}=0$, we see $\kappa_{p}=0$, which is a contradiction. Thus we have $\rho_{p} \neq 0$ and obtain $\alpha_{i}=\delta_{p}+\left(\kappa_{p} / \rho_{p}\right)$. We hence have $\alpha_{1}=\cdots=\alpha_{2 n-2}\left(=\alpha_{p}\right)$. This shows that all tangent vectors at $p$ which are orthogonal to $\xi_{p}$ are principal. Due to the classification of homogeneous Hopf real hypersurfaces in $\mathbb{C} P^{n}$ by Takagi [16], we find that $M$ is locally congruent to a geodesic sphere. Thus the third condition shows that the radius is $r=(2 / \sqrt{c}) \sin ^{-1} \sqrt{2 / 3}$.

In order to study more about geodesic spheres of special radius, we need to recall principal curvatures of homogeneous real hypersurfaces in $\mathbb{C} P^{n}(c)$. Such real hypersurfaces are classified by Takagi [16]. A homogeneous real hypersurface in $\mathbb{C} P^{n}(c)$ is congruent to one of the following: 
1) a geodesic sphere $G(r)$ of raius $r(0<r<\pi / \sqrt{c})$,

2) a tube $T_{\ell}(r)$ of radius $r(0<r<\pi / \sqrt{c})$ around totally geodesic $\mathbb{C} P^{\ell}(1 \leq \ell \leq n-2)$,

3) a tube $Q(r)$ of radius $r(0<r<\pi /(2 \sqrt{c}))$ around complex hyperquadric $\mathbb{C} Q^{n-1}$,

4) tubes of radius $r(0<r<\pi /(2 \sqrt{c}))$ around $\mathbb{C} P^{1} \times \mathbb{C} P^{(n-1) / 2}, G_{2,5}(\mathbb{C})$ and $S O(10) / U(5)$.

The principal curvature $\delta_{M}$ corresponding to $\xi$ is $\delta_{M}=\sqrt{c} \cot \sqrt{c} r$. When $M=T_{\ell}(r)$, it has two principal curvatures

$$
\lambda_{M}^{(1)}=(\sqrt{c} / 2) \cot (\sqrt{c} r / 2) \text { and } \lambda_{M}^{(2)}=-(\sqrt{c} / 2) \tan (\sqrt{c} r / 2)
$$

for tangent vectors orthogonal to $\xi$. When $M=Q(r)$, it also has two principal curvatures

$$
\lambda_{M}^{(1)}=\frac{\sqrt{c}}{2} \cot \left(\frac{\sqrt{c}}{2} r-\frac{\pi}{4}\right) \quad \text { and } \quad \lambda_{M}^{(2)}=\frac{\sqrt{c}}{2} \cot \left(\frac{\sqrt{c}}{2} r+\frac{\pi}{4}\right)
$$

for tangent vectors orthogonal to $\xi$. For other homogeneous real hypersurfaces, they have four principal curvatures

$$
\begin{array}{ll}
\lambda_{M}^{(1)}=\frac{\sqrt{c}}{2} \cot \left(\frac{\sqrt{c}}{2} r-\frac{\pi}{4}\right), & \lambda_{M}^{(2)}=\frac{\sqrt{c}}{2} \cot \left(\frac{\sqrt{c}}{2} r+\frac{\pi}{4}\right), \\
\lambda_{M}^{(3)}=\frac{\sqrt{c}}{2} \cot \frac{\sqrt{c} r}{2}, & \lambda_{M}^{(4)}=-\frac{\sqrt{c}}{2} \tan \frac{\sqrt{c} r}{2}
\end{array}
$$

for tangent vectors orthogonal to $\xi$. It is known that a Hopf real hypersurface all of whose principal curvatures are constant in $\mathbb{C} P^{n}$ is homogeneous.

Theorem 3. A connected real hypersurface $M$ in a complex projective space $\mathbb{C} P^{n}(c)$ of constant holomorphic sectional curvature $c$ is locally congruent to the geodesic sphere of radius $r=(2 / \sqrt{c}) \sin ^{-1} \sqrt{2 / 3}$ if and only if it satisfies the following conditions with some constant $\kappa$ with $|\kappa| \neq \sqrt{2 c} / 4$ at each point $p \in M$ :

i) The condition (ET) holds;

ii) There exist linearly independent tangent vectors $v_{1}, \ldots, v_{2 n-2} \in U_{p} M$ satisfying that the extrinsic shapes of trajectories $\gamma_{i}(i=1, \ldots, 2 n-2)$ for $\mathbb{F}_{\kappa}$ with $\dot{\gamma}_{i}(0)=v_{i}$ are circles of geodesic curvature $\sqrt{2 c} / 4$ and of complex torsion $\tau_{i} \neq \pm 1$.

Proof. We are enough to show the "if" part. By the first condition we see $M$ is a Hopf hypersurface. We denote by $\delta_{M}$ the principal curvature associated with $\xi$. We put $\rho_{i}=$ $\left\langle v_{i}, \xi_{p}\right\rangle$. As $|\kappa| \neq \sqrt{2 c} / 4$, we find along the same lines as of the proof of Theorem 2 that each $v_{i}-\rho_{i} \xi_{p}$ is principal and that $\alpha_{i} \rho_{i}=\delta_{M} \rho_{i}+\kappa$ with the principal curvature $\alpha_{i}$ of $v_{i}-\rho_{i} \xi_{p}$. Moreover, (5.3) shows

$$
c / 8=\kappa^{2}\left(1-\rho_{i}^{2}\right)+\left\{\alpha_{i}+\left(\delta_{M}-\alpha_{i}\right) \rho_{i}^{2}\right\}^{2} .
$$

When $\rho_{i}=0$, we have $\kappa=0$. (5.9) shows that $\alpha_{i}= \pm \sqrt{2 c} / 4$. When $\rho_{i} \neq 0$, substituting $\alpha_{i}=\delta_{M}+\left(\kappa / \rho_{i}\right)$ into (5.9), we find that $\rho_{i}$ satisfies the following equation

$$
2 \kappa \delta_{M} \rho_{i}^{3}+\left\{(c / 8)+\kappa^{2}-\delta_{M}\right\} \rho_{i}^{2}-2 \kappa \delta_{M} \rho_{i}-\kappa^{2}=0 .
$$

Thus $\rho_{i}$ is one of the three solutions of this cubic equation. Therefore, by perturbation theory ([9]) we find that each $\alpha_{i}$ is locally constant. This means that $M$ is a Hopf real hypersurface all of whose principal curvatures are constant. 
We shall check that homogeneous real hypersurfaces except geodesic spheres of radius $r=(2 / \sqrt{c}) \sin ^{-1} \sqrt{2 / 3}$ do not satisfy the second condition. We take $v_{1}, \ldots, v_{2 n-2} \in U_{p} M$ as in the second condition. By the above argument, we see that $v_{i}-\rho_{i} \xi_{p}$ with $\rho_{i}=\left\langle v_{i}, \xi_{p}\right\rangle$ is principal. We denote by $\lambda_{i}$ the principal curvature of $v_{i}-\rho_{i} \xi_{p}$. As $v_{1}-\rho_{1} \xi_{p}, \ldots, v_{2 n-2}-$ $\rho_{2 n-2} \xi_{p}$ span the tangent space $T_{p}^{0} M$ orthogonal to $\xi_{p}$, these $\lambda_{1}, \ldots, \lambda_{2 n-2}$ are all the principal curvatures for $T_{p}^{0} M$. Let $\gamma_{i}$ denote the trajectory for $\mathbb{F}_{K}$ with $\dot{\gamma}_{i}(0)=v_{i}$. Since the complex torsion $\tau_{i}$ of the extrinsic shape of $\gamma_{i}$ is not \pm 1 , by (5.1), (5.3) and by the same computation as for Lemma 1 we have

$$
\begin{aligned}
& \kappa+\left(\delta_{M}-\lambda_{i}\right) \rho_{i}=0, \\
& c / 8=\kappa^{2}-2 \lambda_{i} \kappa \rho_{i}+\lambda_{i}^{2}, \\
& \tau_{i}=4\left(2 \kappa \rho_{i}^{2}-\kappa-\lambda_{i} \rho_{i}\right) / \sqrt{2 c} .
\end{aligned}
$$

When $M=G(r)$ with $r \neq(2 / \sqrt{c}) \sin ^{-1} \sqrt{2 / 3}$, we see in $\S 4$ that if the extrinsic shape of an extrinsic circular trajectory has geodesic curvature $\sqrt{2 c} / 4$ then its complex torsion is \pm 1 , hence it does not satisfy the second condition.

When $M=T_{\ell}(r)$ we have $\delta_{M}=\lambda_{M}^{(1)}+\lambda_{M}^{(2)}, \lambda_{M}^{(2)}=-c /\left(4 \lambda_{M}^{(1)}\right)$, in particular one of $\lambda_{M}^{(1)}, \lambda_{M}^{(2)}$ is not $\sqrt{2 c} / 4$. For a circular trajectory $\gamma_{i}$ corresponding to $\lambda_{i} \neq \sqrt{2 c} / 4$ we have $\tau_{i}^{2}=1$ by the same computation for (4.2). This is a contradiction. Hence $T_{\ell}(r)$ does not satisfies the second condition. Similarly, when $M$ is one of tubes around $\mathbb{C} P^{1} \times \mathbb{C} P^{(n-1) / 2}, G_{2,5}(\mathbb{C})$ and $S O(10) / U(5)$, then $\lambda_{M}^{(3)}, \lambda_{M}^{(4)}$ satisfy $\delta_{M}=\lambda_{M}^{(3)}+\lambda_{M}^{(4)}, \lambda_{M}^{(4)}=-c /\left(4 \lambda_{M}^{(3)}\right)$. Hence we can conclude that it does not satisfy the second condition neither.

When $M=Q(r)$, we have

$$
\lambda_{M}^{(1)}=(\sqrt{c} / 2) v, \quad \lambda_{M}^{(2)}=-(\sqrt{c} / 2) v^{-1}, \quad \delta_{M}=-2 \sqrt{c} /\left(v-v^{-1}\right)
$$

with $v=\cot (\sqrt{c} r / 2)$. When $\lambda_{i}=\lambda_{M}^{(1)}$, we have $\kappa=\sqrt{c}\left(v^{2}+3\right) \rho_{i} /\left\{2\left(v-v^{-1}\right)\right\}$. Substituting this into (5.11) we find

$$
\kappa^{2}=c\left(2 v^{2}-1\right)\left(v^{2}+3\right) /\left\{8\left(v^{2}-5\right)\right\} .
$$

When $\lambda_{i}=\lambda_{M}^{(2)}$, we have $\kappa=\sqrt{c}\left(3+v^{-2}\right) \rho_{i} /\left\{2\left(v-v^{-1}\right)\right\}$. Substituting this into (5.11) we find

$$
\kappa^{2}=c\left(v^{2}-2\right)\left(3 v^{2}+1\right) /\left\{8 v^{2}\left(5 v^{2}-1\right)\right\} .
$$

Comparing these we obtain

$$
0=v^{2}\left(5 v^{2}-1\right)\left(2 v^{2}-1\right)\left(v^{2}+3\right)-\left(v^{2}-5\right)\left(v^{2}-2\right)\left(3 v^{2}+1\right)=10\left(v^{2}+1\right)^{3}\left(v^{2}-1\right) .
$$

Since $0<r<\pi /(2 \sqrt{c})$, we have $v>1$. Hence, the above is a contradiction. Thus $Q(r)$ does not satisfies the second condition. 


\section{References}

[1] T. Adachi: Kähler magnetic flows on a manifold of constant holomorphic sectional curvature, Tokyo J. Math. 18 (1995), 473-483.

[2] T. Adachi: Lamination of the moduli space of circles and their length spectrum for a non-flat complex space form, Osaka J. Math. 40 (2003), 895-916.

[3] T. Adachi: Trajectories on geodesic spheres in a non-flat complex space form, J. Geom. 90 (2008), 1-29.

[4] T. Adachi: Extrinsic circular trajectories on geodesic spheres in a complex projective space, in Differential Geometry of Submanifolds and its Related Topics, S. Maeda, Y. Ohnita and Q.-M. Cheng, eds, World Scientific Publ. Singapore (2013), 44-59.

[5] T. Adachi and S. Maeda: Length spectrum of circles in a complex projective space, Osaka J. Math. 35 (1998), 553-565.

[6] T. Adachi, S. Maeda and S. Udagawa: Circles in a complex projective space, Osaka J. Math. 32 (1995), 709-719.

[7] T. Bao and T. Adachi: Circular trajectories on real hypersurfaces in a nonflat complex space form, J. Geom. 96 (2009), 41-55.

[8] A. Comtet: On the Landau levels on the hyperbolic plane, Ann. Phys. 173 (1987), 185-209.

[9] T. Kato: Perturbation Theory for linear operators, Springer-Verlag, 1966.

[10] Y. Maeda: On real hypersurfaces of a complex projective space, J. Math. Soc. Japan 28 (1976), 529-540.

[11] S. Maeda and T. Adachi: Sasakian curves on hypersurfaces of type (A) in a nonflat complex space form, Results Math. 56 (2009), 489-499.

[12] S. Maeda, T. Adachi and Y.H. Kim: Characterizations of geodesic hyperspheres in a non-flat complex space form, Glasgow Math. J. 55 (2013), 217-227.

[13] S. Maeda and Y. Ohnita: Helical geodesic immersion into complex space form, Geom. Dedicata 30 (1989), 93-114.

[14] H. Naitoh: Isotropic submanifolds with parallel second fundamental form in $P^{m}(\mathbb{C})$, Osaka J. Math. 18 (1981), 427-464.

[15] T. Sunada: Magnetic flows on a Riemann surface, Proc. KAIST Math. Workshop 8 (1993), 93-108.

[16] R. Takagi: Real hypersurfaces in a complex projective space with constant principal curvatures, J. Math. Soc. Japan 27 (1975), 43-53.

[17] A. Weinstein: Distance spheres in complex projective spaces, Proc. Amer. Math. Soc. 39 (1973), 649-650.

Tuya Bao

College of Mathematics

Inner Mongolia University for the Nationalities

Tongliao, Inner Mongolia, 028043

People's Republic of China

e-mail: baotuya1981@126.com

Toshiaki Adachi

Department of Mathematics

Nagoya Institute of Technology

Nagoya 466-8555

Japan

e-mail: adachi@nitech.ac.jp 